\title{
Microemulsion model with oil-water anisotropy
}

\author{
Marcia C. Barbosa and Marcos Frichembruder \\ Instituto de Física, Universidade Federal do Rio Grande do Sul, Caixa Postal 15051, CEP 91501-970, Porto Alegre, \\ Rio Grande do Sul, Brazil
}

(Received 11 August 1994; revised manuscript received 3 January 1995)

\begin{abstract}
We consider a spin model for applications to oil-water-amphiphilic-surfactant mixtures near the region where those phases coexist. We analyze this model assuming that oil and water molecules cannot be treated symmetrically, given that they do exhibit different chemical potentials. Using a mean-field approximation, we find that the modulated phase assumes two possible arrangements, such as sheets (lamellar phase) or rods (hexagonal phase). Due to fluctuations, the lamellar phase is present when the difference between the chemical potential of oil and the chemical potential of water is not too high. Both lamellar and hexagonal phases are present when this difference exceeds a certain value. This value specifies the triple point where the two modulated phases coexist with the disordered phase. This point is present even for small values of the critical wave vector, indicating that the mean-field Lifshitz point is actually a fluctuation-induced end point.
\end{abstract}

PACS number(s): 82.70.Kj, 05.70.Fh, 64.60.Ak, 64.60.Cn

\section{INTRODUCTION}

Systems of oil, water, and amphiphilic surfactant exhibit many interesting properties both from the practical and from the theoretical point of view. This made them subject to a great deal of experimental and theoretical studies [1].

The experimental observations can be summarized as follows [2]. At low temperatures, on mixing oil, water, and a surfactant, one finds that, besides the oil-rich and water-rich phases (phases where water and oil do not $\mathrm{mix}$ ), the system also exhibits an isotropic phase where oil regions and water regions are separated by layers of amphiphilic molecules. This phase, called microemulsion, exhibits nonmonotonically decaying correlation functions, and it can be identified as a structured fluid which is homogeneous on large length scales, but remains heterogeneous over small length scales [3].

For a large concentration of surfactant, a transition from the disordered structured phase to an ordered structured phase is observed. This modulated phase can have many different kind of arrangements, such as lamellar (sheets of amphiphiles separating oil-rich from water-rich regions) or hexagonal (rods of amphiphiles separating oilrich from water-rich regions) phases [2].

At high temperatures and small concentrations of surfactant, the usual disordered fluid is separated from the disordered structured fluid, the microemulsion, by a Lifshitz line. This line is quite interesting for itself, since it is the locus where the peak of the water-water correlation function moves away from zero wave vector without a phase transition [4]. Figure 1 illustrates, in schematic form, the phase diagram [1].

In some specific systems, as one proceeds from the phase where oil and water coexist to the microemulsion region, a state where those three phases coexist is found. It is the so called Winsor III state [4]. This state is quite controversial. It is also called a bicontinuous phase, since it exhibits a sudden increase in the magnitude of its electrical conductivity. In some systems, a sharp variation in its dielectric behavior and in its viscosity, as the temperature reaches a limit, is also observed [5]. The oil-watermicroemulsion coexisting region exhibits also an ultralow surface tension between those phases [6].

Even if the above results seem to indicate that the microemulsion is almost critical, given that the volume fraction of each component differs and that no wetting phenomena are observed, we might suspect that no critical phenomenon happens.

From the practical point of view, since oil and water are not soluble at room temperature, the very existence of the Winsor III state is interesting. Thus it seems a challenge to be able to describe all those phenomena within a simple model. In that sense, many different kind of models have been proposed [7-11]. Most of them, rather than catch all the details, try to retain only the essential features of those mixtures. Particularly, some of them sim-

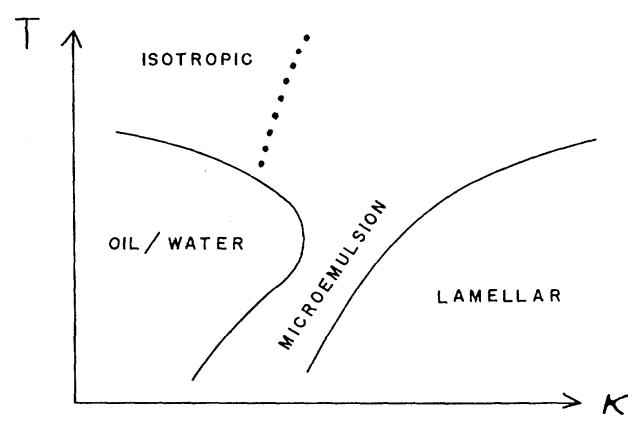

FIG. 1. Phase diagram in terms of temperature $T$ and $\kappa_{2} / 2=\kappa_{1}=\kappa$, for oil-water-amphiphilic systems. First-order transitions separate the oil-rich and water-rich coexisting regions from the isotropic fluid phase and the lamellar phase from the microemulsion region. This diagram shows schematically results from simulations and experiments. 


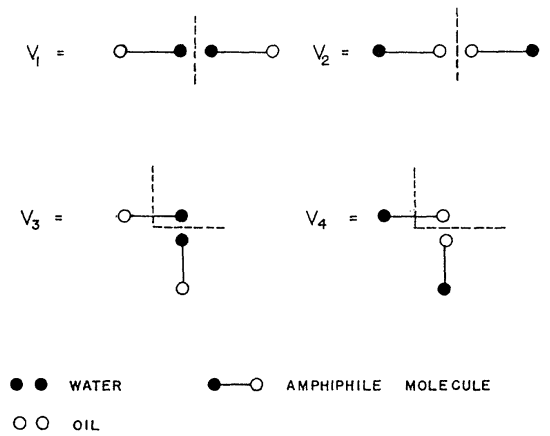

FIG. 2. Different types of configurations of a pair of amphiphiles.

ply employ a lattice, where the oil, water, or amphiphilic molecules are restricted to occupy only the sites of the lattice rather than any point of the space.

This paper discusses the phase diagram of one of those models: the Ising model with next-nearest-neighbor isotropic competing interactions, which we will call the INNNI model. In this model, introduced by Widom [7] and generalized by Upton and Yeomans [12], the space configuration is divided into cubes. These cubes are linked by oil $(A A)$, water $(B B)$, or amphiphilic $(A B)$ molecules (see Fig. 2). This is done with the constraint that only the same type of ends $(A$ or $B)$ of different molecules is permitted within each cube. Consequently, one amphiphilic molecule just connects two different cubes and acts as an interaction term, what leads ultimately to an Ising model.

This model was studied before, assuming that oil and water exhibit the same chemical potential, and that the amphiphilic molecule is symmetric (here we represented that by taking $h=0$, as we will see later). Within the mean-field approximation, the phase-diagram for $h=0$ goes as follows. One continuous phase transition separates the disordered phase from the usual ordered phase (oil-rich phase or water-rich phase). Another continuous transition separates the structured disordered phase from

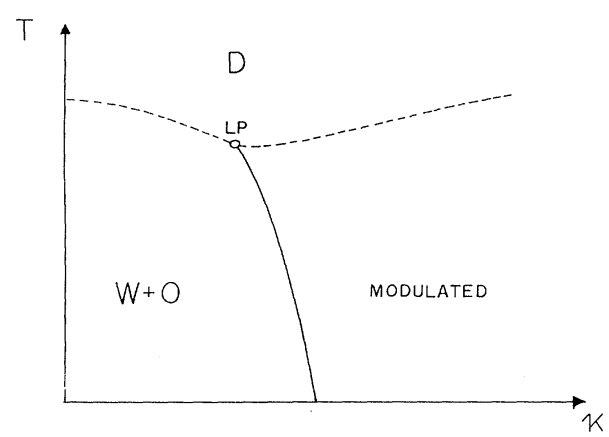

FIG. 3. Schematic mean-field phase diagram of the three-dimensional Widom model for a microemulsion in terms of temperature, $\kappa_{2} / 2=\kappa_{1}=\kappa$, and $h=0$. The solid line represents the first-order transition between the oil-rich phase and the modulated (lamellar) phase. The dashed lines are the continuous transtions. Those lines meet at the Lifshitz point LP. the modulated phase, the lamellar phase. A first-order phase boundary line is found between the lamellar and the oil-rich (or water-rich) phases. The two critical lines meet the first-order phase boundary at a Lifshitz point (see Fig. 3).

Including fluctuations, one finds that the transition from the disordered to the modulated phase is actually first order. This result was obtained both in the lattice model $[13-15]$ and in its continuous version $[16,17]$. The absence of a continuous transition between the disordered phase and the modulated phase leads to a controversy about the character of the Lifshitz point. Three possible scenarios were suggested [see Figs. 4(a)-4(c)]. One possi-
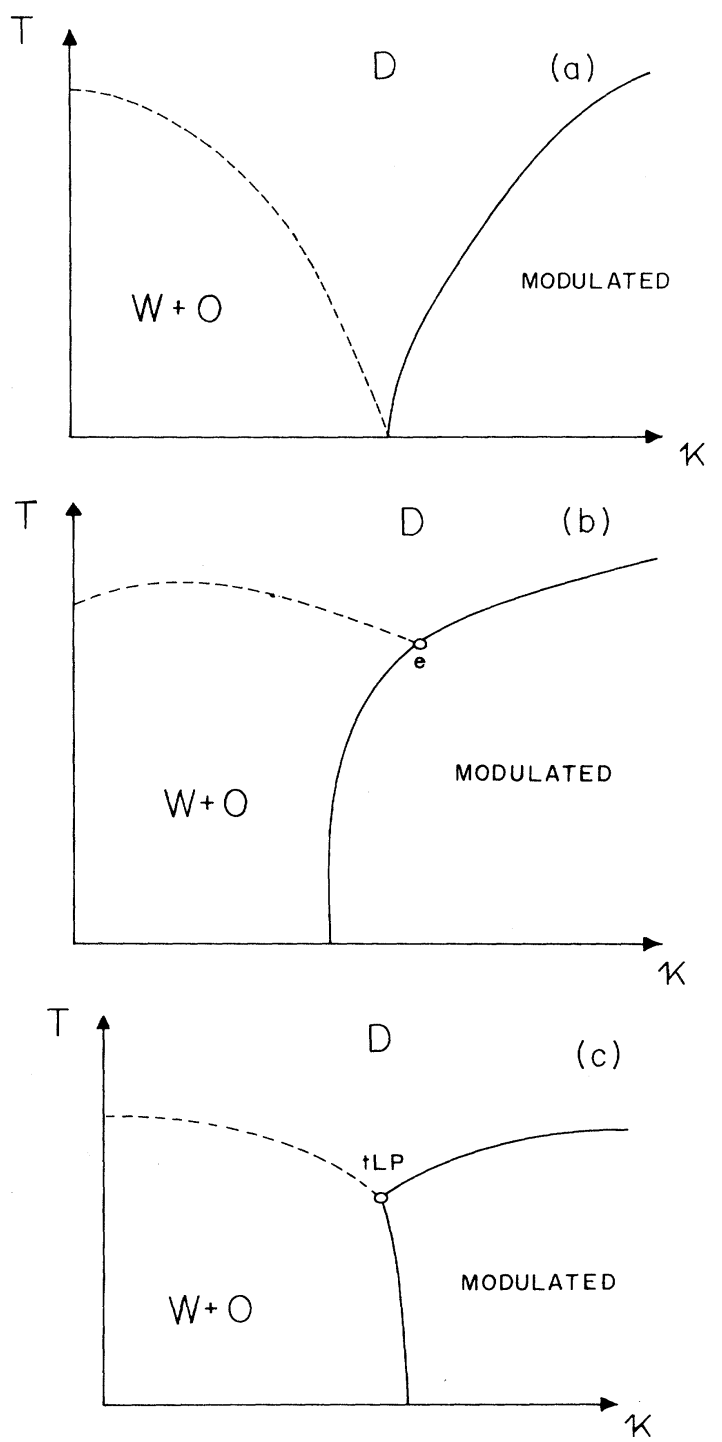

FIG. 4. Possible scenarios for the phase diagram near the Lifshitz point. Solid lines represent first-order transitions, while dashed lines represent continuous transitions. (a) There is no phase boundary between the ordered phase and the modulated phase. (b) The disordered-ordered continuous line ends at an end point. (c) The Lifshitz point is really a tricritical Lifshitz point (tLP). 
bility is that there should be no phase transition between the ordered phase and the modulated phase and that the first-order phase boundary between the microemulsion phase and modulated phase should come to an end at $T=0$ [see Fig. 4(a)] [15]. Another possibility is that the continuous transition line (ordered-disordered line) meets the two first-order lines (disordered-modulated and ordered-modulated lines) at an end point. In that case, both first-order phase boundary lines should share the same tangent and, consequently, fluctuations should induce a change in the phase boundary between the two ordered phases [see Fig. 4(b)] [14]. Finally, another possibility is that the Lifshitz point should be a tricritical point [see Fig. 4(c)]. This is the case if the phase separation between the microemulsion and ordered structured phase becomes critical when the critical wave vector vanishes [18-20].

In the present work, we try to understand what does happen near the Lifshitz point. In order to do that, we assume that oil and water exhibit different chemical potentials $\left(h \propto \mu_{w}-\mu_{o} \neq 0\right.$ where $\mu_{w}$ and $\mu_{o}$ are the chemical potentials of water and oil), and that there is
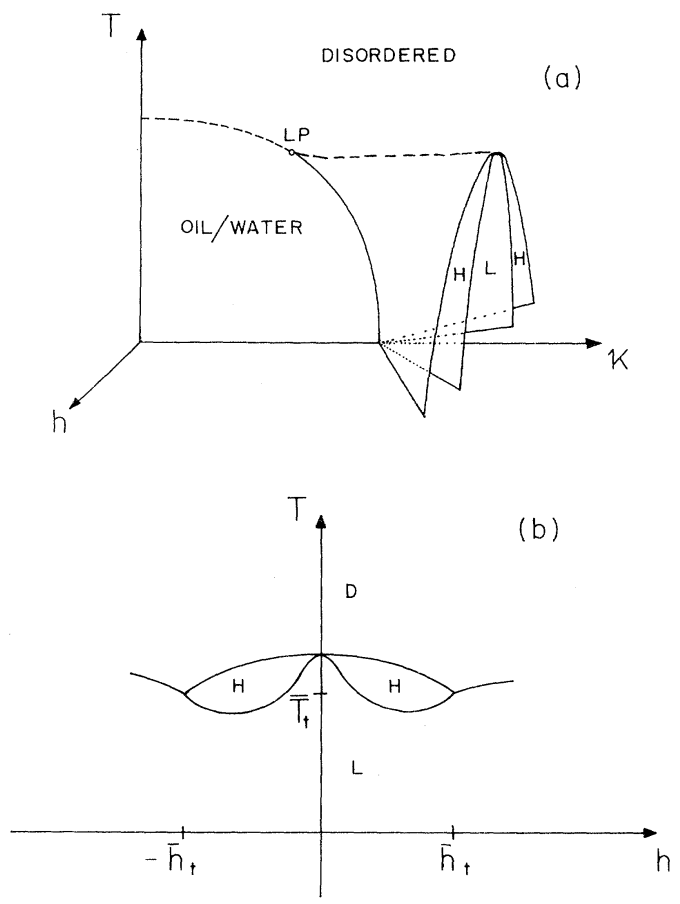

FIG. 5. (a) Schematic mean-field phase diagram of the three-dimensional Widom model for a microemulsion in terms of temperature $T, \kappa_{2} / 2=\kappa_{1}=\kappa$, and ordering field $h$. The figure shows the region where $T \ll \bar{T}_{t}$ where the disordered phase is separated from the oil-rich or water-rich phases by a continuous transition (long-dashed line). The point LP locates the Lifshitz point. A first-order surface separates the disordered phase from both modulated phases, hexagonal $(H)$ and lamellar $(L)$ phases. (b) The phase diagram for fixed $\kappa$ and general $T$. For $T=\tilde{T}_{t}$ there is a triple point where the lamellar-disordered phase boundary meets the hexagonal-disordered first-order line and the lamellar-hexagonal separation line. a different energy cost between bending the amphiphilic film around a molecule of water and bending it around a molecule of oil.

Given that, we obtain, within mean-field approximation, the following phase diagram (see Fig. 5). There are three possible nonstructured phases: disordered, lamellar, and hexagonal phases. For $0<h<\bar{h}_{t}$ and at the high temperature region, the disordered phase coexists with the hexagonal phase along a first-order phase boundary. Lowering the temperature, one finds a firstorder transition between two different types of modulated phases: hexagonal phase and lamellar phase. For $h>\bar{h}_{t}$ and at high temperatures, the lamellar phase coexists with the disordered phase since no hexagonal phase is found. At $\left(\bar{T}_{t}, \bar{h}_{t}\right)$ the two modulated phases coexist with the disordered phase at a triple point. On decreasing the concentration of surfactant, varying the critical wave vec-
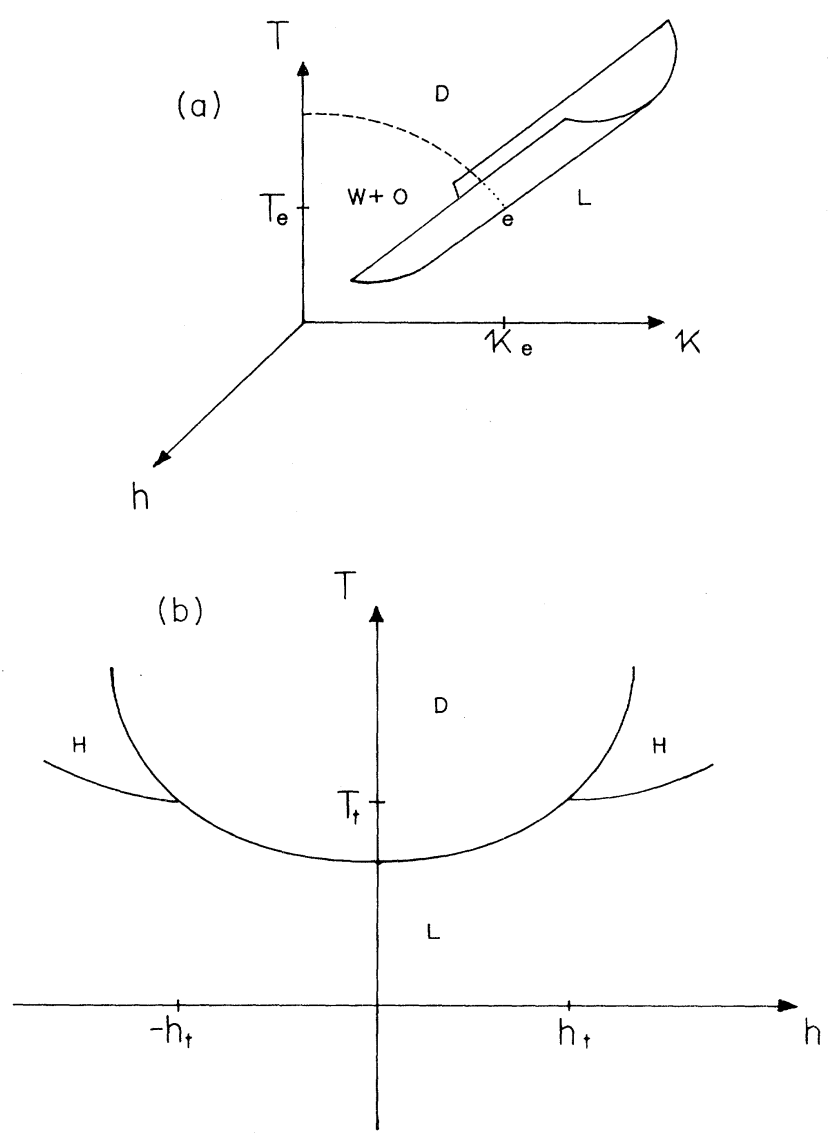

FIG. 6. (a) Schematic phase diagram of the three-dimensional Widom model for a microemulsion in terms of temperature, $\kappa$, and ordering field $h$, assuming $T<T_{t}$. The disordered phase is separated from the oil-rich or water-rich phases by a continuous phase transition (long-dashed line). This line meets the first order phase boundary between the disordered phase $(D)$ and lamellar phase $(L)$ and between the nonstructured ordered phase $(O+W)$ and the lamellar phase at an end point $e$. (b) The phase diagram for a fixed $\kappa$ and general $T$. At $\left(h_{t}, T_{t}\right)$, there is a triple point where the lamellar-disordered, lamellar-hexagonal, and hexagonal-disordered lines meet. 
tor $\left(q_{c} \rightarrow 0\right)$, the region where the hexagonal phase is found decreases in size. The first-order phase boundary lines collapse at $\left(h=0, q_{c}=0\right)$ at the Lifshitz point (see Figs. 3 and 5).

We then check the above results against fluctuations. Using a harmonic approximation and a diagrammatic expansion, we show that the transition between the disordered phase and the modulated phase is first order for zero and nonzero external fields $[16,17]$. In the presence of a small external field, $h<h_{t}$, the transition between the disordered and the ordered structured phases is first order. The modulated phase, in that case, is always lamellar [see Fig. 6(a)]. As one increases the external field, for $h>h_{t}$, besides the lamellar (present for $T<T_{h}$ ), a hexagonal phase also can be found (for $T>T_{h}$ ). A phase boundary line between those two modulated phases is found for $T=T_{h}$ [see Fig. 6(b)]. The disordered phase, the lamellar phase, and the hexagonal phase meet at a triple point at $\left(h_{t}, T_{t}\right)$. On varying the parameter related to the bending energy cost of the amphiphilic film, a triple line is generated. This line is a nondecreasing function of the critical wave vector, indicating that the Lifshitz point should be an end point.

The article is organized as follows. In Sec. II, the model is introduced and the mean-field phase diagram is found. In Sec. III, we obtain an expression for the equation of state when fluctuations are allowed. Conclusions end each section.

\section{MODEL}

Let us considered the following model. Each simple cubic lattice site $\vec{r}$ is occupied by an Ising spin $s(\vec{r})$ that interacts through nearest-neighbor interactions of strength $J$, next-nearest-neighbor couplings along the cubic axes of strength $-\kappa_{1} J$, and next-nearest-neighbor interactions across the face diagonals, $-\kappa_{2} J$. The Hamiltonian of such a system is given by

$$
\mathcal{H}=-\frac{1}{2} \sum_{\vec{r}, \overrightarrow{r_{1}}} s(\vec{r}) J\left(\vec{r}-\overrightarrow{r_{1}}\right) s\left(\overrightarrow{r_{1}}\right)-H \sum_{\vec{r}} s(\vec{r}),
$$

where $H$ is here a homogeneous external field.

This Hamiltonian describes a microemulsion system as follows [7]. Let us assume a configuration space, divided into cubes. Each cube is occupied by oil $(A A)$, water $(B B)$, and amphiphilic $(A B)$ molecules subject to the constraint that only like ends of different molecules can occupy the same cube (see Fig. 2). In this sense, each cube of the configurational space can be in two different states, oil or water, that we can represent by Ising spins $s=-1$ and $s=+1$, respectively. The interface between oil and water is then occupied by a film of amphiphilic molecules. Such kind of films must have a positive curvature energy, tending to keep it flat, thus producing large oil-coherent and water-coherent regions. This is done by introducing positive interaction energy terms $\left\{V_{i}\right\}$ (see Fig. 2). We will assume that $V_{1}$ and $V_{2}$ are the energies of parallel amphiphilic molecules, while $V_{3}$ and $V_{4}$ are the energies of two molecules at an angle. Note that, due to the asymmetry within the amphiphilic molecule, the energy of the water-oil interface is not the same energy of the oil-water interface. We represent this fact by assuming that $V_{1} \neq V_{2}$ and that $V_{3} \neq V_{4}$. Particularly, we will take that $V_{1}+V_{2}=\left(V_{3}+V_{4}\right)$.

Using the parameters $V_{i}$ and the chemical potentials of the water, $\mu_{A A}$, the oil, $\mu_{B B}$, and the amphiphilic molecules, $\mu_{A B}$, one can write a partition function for the microemulsion model $[1,7]$. Comparing this partition function with the partition function associated with the INNNI model [see Eq. (1)], one finds that they are equivalent. Table I gives the relation between the parameters of the Ising model and the parameters of the microemulsion model $[1,7]$.

Note that, if $V_{1}+V_{2}=\left(V_{3}+V_{4}\right)$, one has that the equality $\kappa \equiv \kappa_{1}=\kappa_{2} / 2$ holds in the Ising model. Assuming that $J$ is fixed, one finds that the bending energy of the amphiphilic film, $\kappa$, is proportional to the chemical potential $\Delta \mu \equiv \mu_{A B}-1 / 2\left(\mu_{A A}+\mu_{B B}\right)$. This last expression is related to the concentration of amphiphiles.

As usual [12-19], the mean-field free energy associated with this model is derived from the Bogoliubov inequality and is given by

$$
\begin{aligned}
F= & N^{-1} \sum_{\vec{r}}\left\{-k_{B} T \ln \left[2 \operatorname { c o s h } \left(\sum_{\overrightarrow{r_{1}}} \beta J\left(\vec{r}-\vec{r}_{1}\right) m\left(\vec{r}_{1}\right)\right.\right.\right. \\
& \left.+\beta H)]+\frac{1}{2} \sum_{\vec{r}_{1}} m(\vec{r}) J\left(\vec{r}-\vec{r}_{1}\right) m\left(\vec{r}_{1}\right)\right\}
\end{aligned}
$$

where $m(\vec{r})=\langle s(\vec{r})\rangle_{0}$ is the magnetization $\left(\langle\rangle_{0}\right.$ is the ensemble average with respect to the trial Hamiltonian in the Bogoliubov inequality) specified by

$$
m(\vec{r})=\tanh \left[\beta \sum_{\vec{r}_{1}} J\left(\vec{r}-\vec{r}_{1}\right) m\left(\vec{r}_{1}\right)+\beta H\right] .
$$

At high temperatures, this equation is fulfilled by a nonzero paramagnetic solution given by

$$
m_{0}=\tanh \left[\beta H+\beta m_{0} \sum_{\overrightarrow{r_{1}}} J\left(\vec{r}-\vec{r}_{1}\right)\right]
$$

and, consequently, it is convenient to write the free energy in terms of

$$
\tilde{m}(\vec{r})=\beta \sum_{\vec{r}_{1}} J\left(\vec{r}-\vec{r}_{1}\right)\left[m\left(\overrightarrow{r_{1}}\right)-m_{0}\left(\overrightarrow{r_{1}}\right)\right]
$$

TABLE I. Relation between the Ising variables and the microemulsion variables corresponding to the interactions given in Fig. 1.

\begin{tabular}{c|l}
\hline \hline Ising Variables & \multicolumn{1}{|c}{ Microemulsion Variables } \\
\hline$H$ & $3 / 2\left(\mu_{B B}-\mu_{A A}\right)+3 / 8\left(V_{1}-V_{2}\right)+3 / 2\left(V_{3}-V_{4}\right)$ \\
$J$ & $1 / 4\left(V_{1}+V_{2}\right)+V_{3}+V_{4}$ \\
$-1 / 2\left[\mu_{A B}-1 / 2\left(\mu_{B B}+\mu_{A A}\right)\right]$ \\
& $1 / 8\left(V_{1}+V_{2}\right)$ \\
$\kappa_{1} J$ & $1 / 4\left(V_{3}+V_{4}\right)$ \\
$\kappa_{2} J$ & \\
\hline
\end{tabular}


Now, close to criticality, the magnetization $\tilde{m}$ is small. Consequently, one can linearize Eq. (2) and the following free energy is obtained:

$$
\begin{aligned}
F= & F_{0}+\frac{1}{2} \sum_{\vec{q}} \tilde{m}(\vec{q}) \tilde{r}(\vec{q}) \tilde{m}(-\vec{q}) \\
& +\frac{w}{3 !} \sum_{\left\{\vec{q}_{i}\right\}} \tilde{m}\left(\vec{q}_{1}\right) \tilde{m}\left(\vec{q}_{2}\right) \tilde{m}\left(\vec{q}_{3}\right) \delta\left(\sum_{i} \vec{q}_{i}\right) \\
& +\frac{u}{4 !} \sum_{\left\{\vec{q}_{i}\right\}} \tilde{m}\left(\vec{q}_{1}\right) \tilde{m}\left(\vec{q}_{2}\right) \tilde{m}\left(\vec{q}_{3}\right) \tilde{m}\left(\overrightarrow{q_{4}}\right) \delta\left(\sum_{i} \vec{q}_{i}\right) \\
& -\sum_{\vec{q}} \tilde{h}(-\vec{q}) \tilde{m}(\vec{q}) .
\end{aligned}
$$

Here $F_{0}$ is independent of $\tilde{m}(\vec{q})$ and $m_{0}$ is given by the zero of $\tilde{h}$. The parameters in Eq. (6) are given by

$$
\begin{aligned}
\tilde{h}(\vec{q}) & =\left(H-\left[k_{B} T-J(\vec{q})\right] m_{0}-k_{B} T \frac{m_{0}^{3}}{3}\right) \delta(\vec{q}), \\
\tilde{r}(\vec{q}) & =r(\vec{q})+\frac{u}{2} m_{0}^{2} \\
r(\vec{q}) & =k_{B} T\left[\frac{k_{B} T}{J(\vec{q})}-1\right] \\
w & =2 k_{B} T m_{0} \\
u & =2 k_{B} T .
\end{aligned}
$$

Analyzing the free energy Eq. (6) and Eq. (7), one finds that the disordered phase is separated from the ordered phase by a critical surface specified by

$$
k_{B} T_{c}=\max _{\vec{q}}\left\{J(\vec{q})\left(1-m_{0}^{2}\right)\right\}
$$

where $q_{c}$ specifies the critical wave vector. Now, if $1-$ $12 \kappa>0$ (here we are assuming that $\kappa \equiv \kappa_{1}=\kappa_{2} / 2$ ), the critical wave vector is zero and the ordered phase is ferromagnetic. If, otherwise, $1-12 \kappa<0$, the ordered phase can be of two types: a lamellar phase given by

$$
\tilde{m}(\vec{r})=2 m_{l} \cos \left(q_{c} z\right),
$$

where planes of oil are followed by planes of water, or a hexagonal phase given by

$$
\begin{aligned}
\tilde{m}(\vec{r})= & 2 m_{h}\left[\cos \left(q_{c} z\right)+\cos \left(\frac{\sqrt{3}}{2} q_{c} y-\frac{1}{2} q_{c} z\right)\right. \\
& \left.+\cos \left(\frac{\sqrt{3}}{2} q_{c} y+\frac{1}{2} q_{c} z\right)\right]
\end{aligned}
$$

where rods of oil are followed by water. Both structures exhibit the same value for the critical wave vector given by

$$
\cos q_{c}=\frac{1-8 \kappa}{4 \kappa} .
$$

It is then clear that the critical modes $\tilde{m}\left(q_{c}\right)$ play the role of the Landau order parameter in this theory. We take the noncritical modes into account by minimization of the free energy, Eq. (6), with respect to $\tilde{m}(q=0), \tilde{m}\left(2 q_{c}\right), \ldots$. Those contributions are, then, eliminated in favor of the critical variables $\tilde{m}\left(q_{c}\right)$, leading to the following free energy for the lamellar phase:

$$
F_{l}=F_{0}+\tilde{r} m_{l}^{2}+\frac{1}{4} u_{l} m_{l}^{4}
$$

where

$$
u_{l}=u-\frac{2 w^{2}}{\tilde{r}(0)}-\frac{w^{2}}{\tilde{r}\left(2 q_{c}\right)} .
$$

Following similar steps, we find that the free energy associated with the hexagonal phase is given by

$$
F_{h}=F_{0}+3 \tilde{r} m_{h}^{2}+2 w m_{h}^{3}+\frac{15}{4} u_{h} m_{h}^{4}
$$

where

$$
u_{h}=u-\frac{6 w^{2}}{5 \tilde{r}(0)}-\frac{w^{2}}{5 \tilde{r}\left(2 q_{c}\right)}
$$

The analysis of these free energies determines that, at high temperatures and low values of the external field, for $h<\bar{h}_{t}$, there is a first-order phase transition between the disordered and hexagonal phases. As one decreases the temperature, there is a first-order transition between the hexagonal and lamellar phases. For high values of the external field, namely, for $h>\bar{h}_{t}$, the coupling $u_{l}$ given by Eq. (13) changes sign and a higher order contribution given by

$$
\Delta F_{l}=\frac{1}{6 !} v_{l} m_{l}^{6}
$$

has to be added to Eq. (12) in order to make the lamellar phase stable. In that case, at high temperatures, the disordered phase coexists with the lamellar phase and the hexagonal phase is not present. At $h=\bar{h}_{t}$, the three phases meet at a triple point [see Fig. 5(b)]. Varying the critical wave vector, one verifies that, as $q_{c} \rightarrow 0$, the region where the hexagonal phase is present disappears. The phase diagram, displayed in terms of the temperature related parameter $r(0) \propto k_{B} T / J$, external field $h$, and the parameter $\kappa$, is shown in Fig. 5(a). Figure 5(b) shows the same diagram for $h=0$.

In summary, the introduction of an external field and the inclusion of noncritical modes have two major effects: the modulated-disorder phase transition changes from continuous to first order and a new modulated phase, the hexagonal phase, is created.

\section{FLUCTUATION EFFECTS}

Now let us consider what does happen when fluctuations are allowed. On going to a continuous representation by adding a weighting term $w(s)=s(\vec{r})^{2} / 2+$ 
$u_{4} s(\vec{r})^{4} / 4$ ! for each spin, one finds a Hamiltonian given by

$$
\begin{aligned}
\overline{\mathcal{H}}= & -\frac{1}{2} \sum_{\vec{q}} s(\vec{q}) u_{2}(\vec{q}) s(-\vec{q}) \\
& -\frac{u_{4}}{4 !} \sum_{\left\{\vec{q}_{i}\right\}} s\left(\vec{q}_{1}\right) s\left(\vec{q}_{2}\right) s\left(\vec{q}_{3}\right) s\left(\vec{q}_{4}\right) \delta\left(\sum_{i} \vec{q}_{i}\right) \\
& +\sum_{\vec{q}} h(-\vec{q}) s(\vec{q}),
\end{aligned}
$$

where, as usual, we have $\overline{\mathcal{H}}=-\mathcal{H} / k_{B} T-w(s)$ with $\mathcal{H}$ given by the Fourier transform of Eq. (1) and where the couplings are given by

$$
\begin{aligned}
u_{2}(\vec{q}) & =1-\frac{J(\vec{q})}{k_{B} T}, \\
h(\vec{q}) & =\frac{H}{k_{B} T} \delta(\vec{q}),
\end{aligned}
$$

where $J(\vec{q})$ is the Fourier transform of $J\left(\vec{r}-\vec{r}_{1}\right)$.

Now, due to the presence of an external field, even in the paramagnetic phase a nonzero magnetization $m_{0}$ is present. Thus it seems natural to shift the spin variables $s(0)=m_{0}+\tilde{s}(0)$ and $s(\vec{q} \neq 0)=\tilde{s}(\vec{q})$, and rewrite the Hamiltonian as

$$
\begin{aligned}
\overline{\mathcal{H}}= & \mathcal{H}_{0}+\tilde{\mathcal{H}}, \\
\tilde{\mathcal{H}}= & -\frac{1}{2} \sum_{\vec{q}} \tilde{s}(\vec{q}) \tilde{r}(\vec{q}) \tilde{s}(-\vec{q}) \\
& -\frac{\tilde{w}}{3 !} \sum_{\left\{\vec{q}_{i}\right\}} \tilde{s}\left(\vec{q}_{1}\right) \tilde{s}\left(\vec{q}_{2}\right) \tilde{s}\left(\vec{q}_{3}\right) \delta\left(\sum_{i} \vec{q}_{i}\right) \\
& -\frac{\tilde{u_{4}}}{4 !} \sum_{\left\{\vec{q}_{i}\right\}} \tilde{s}\left(\vec{q}_{1}\right) \tilde{s}\left(\vec{q}_{2}\right) \tilde{s}\left(\vec{q}_{3}\right) \tilde{s}\left(\vec{q}_{4}\right) \delta\left(\sum_{i} \vec{q}_{i}\right) \\
& +\sum_{\vec{q}} \tilde{h}(-\vec{q}) \tilde{s}(\vec{q}),
\end{aligned}
$$

where $m_{0}$ is specified by $\tilde{h}=0$, and

$$
\begin{aligned}
\tilde{r}(\vec{q}) & =u_{2}(\vec{q})+\frac{1}{2} u_{4} m_{0}^{2} \\
\tilde{w} & =u_{4} m_{0}, \\
\tilde{h}(\vec{q}) & =\left(h-u_{2} m_{0}-\frac{u_{4}}{6} m_{0}^{3}\right) \delta(\vec{q}), \\
\tilde{u_{4}} & =u_{4},
\end{aligned}
$$

and where $\mathcal{H}_{0}$ does not depend on the spin variables.

Now, since we are interested in the Lifshitz region, we will write the parameters in Eq. (18) assuming that the critical wave vector $q_{c}$ is small. Within this approximation, the continuum version of the Hamiltonian Eq. (18) is given by

$$
\begin{aligned}
\tilde{\mathcal{H}}= & -\frac{1}{2} \int d^{3} q \phi(\vec{q}) \tilde{r}(\vec{q}) \phi(\vec{q}) \\
& -w \iiint d^{3} q_{1} d^{3} q_{2} d^{3} q_{3} \phi\left(\vec{q}_{1}\right) \phi\left(\vec{q}_{2}\right) \phi\left(\vec{q}_{3}\right) \delta\left(\sum_{i} \vec{q}_{i}\right) \\
& -u \iiint \int d^{3} q_{1} d^{3} q_{2} d^{3} q_{3} d^{3} q_{4} \phi\left(\vec{q}_{1}\right) \phi\left(\vec{q}_{2}\right) \phi\left(\vec{q}_{3}\right) \phi\left(\vec{q}_{4}\right) \delta\left(\sum_{i} \vec{q}_{i}\right) \\
& +\int d^{3} q \tilde{h}(-\vec{q}) \phi(\vec{q}),
\end{aligned}
$$

where

$$
\begin{aligned}
\tilde{r}(\vec{q}) & =r_{0}+\left(q^{2}-q_{c}^{2}\right)^{2} \\
r_{0} & =\frac{k_{B} T-6 J(1-5 \kappa)}{\kappa k_{B} T}+q_{c}^{4} \\
q_{c}^{2} & =\frac{J}{2 k_{B} T}\left(\frac{1-12 \kappa}{\kappa}\right) \\
w & =\frac{\tilde{w}}{\kappa^{3 / 2}} \\
u & =\frac{u_{4}}{\kappa^{2}}
\end{aligned}
$$

and where $\phi(\vec{q}) \equiv \sqrt{\kappa} \tilde{s}(\vec{q})$.

In order to study the ordered phase, we introduce a field $\phi(\vec{q})=\psi(\vec{q})+\bar{\phi}(\vec{q})$, so that $\langle\phi(\vec{q})\rangle=\bar{\phi}(\vec{q})$ and, consequently, $\langle\psi\rangle=0$ (here, as usual, $\langle a\rangle \equiv \bar{a}$ represents the average of $a$ ). The introduction of an external field $h$ conjugate to $\bar{\phi}(\vec{q})$ given by

$$
h(\vec{q})=\left\langle\frac{\partial \tilde{\mathcal{H}}}{\partial \bar{\phi}(\vec{q})}\right\rangle
$$

leads to the equation of state [21]

$$
\begin{aligned}
h(\vec{q})= & {\left[r_{0}+\left(q^{2}-q_{c}^{2}\right)^{2}\right] \bar{\phi}(\vec{q})+\frac{w}{2} \int d^{3} q_{1} \bar{\phi}\left(\overrightarrow{q_{1}}\right) \bar{\phi}\left(\vec{q}-\overrightarrow{q_{1}}\right) } \\
& +\frac{u}{6} \iint d^{3} q_{1} d^{3} q_{2} \bar{\phi}\left(\overrightarrow{q_{1}}\right) \bar{\phi}\left(\overrightarrow{q_{2}}\right) \bar{\phi}\left(\vec{q}-\overrightarrow{q_{1}}-\overrightarrow{q_{2}}\right) \\
& +\frac{u}{2} \int d^{3} q_{1}\left\langle\psi\left(\overrightarrow{q_{1}}\right) \psi\left(-\overrightarrow{q_{1}}\right)\right\rangle \bar{\phi}(\vec{q}) \\
& +\frac{w}{2} \int d^{3} q_{1}\left\langle\psi\left(\overrightarrow{q_{1}}\right) \psi\left(-\overrightarrow{q_{1}}+\vec{q}\right)\right\rangle,
\end{aligned}
$$


where terms like $\left\langle\psi\left(\overrightarrow{q_{1}}\right) \psi\left(\overrightarrow{q_{2}}\right) \psi\left(\overrightarrow{q_{3}}\right)\right\rangle$ are of lower order [16].

Now one needs to compute $\left\langle\psi\left(\overrightarrow{q_{1}}\right) \psi\left(-\overrightarrow{q_{1}}+\vec{q}\right)\right\rangle$. Since the minimum of $\tilde{r}$ is obtained at $q=q_{c}$, the field amplitudes with $q=q_{c}$ increase fast when $\tilde{r} / q_{c} \rightarrow 0$ and, consequently, the mean square of fluctuations to first order in a loop expansion is given by

$$
\begin{aligned}
\langle\psi(\vec{q}) \psi(-\vec{q})\rangle & =\tilde{r}(q)+\frac{u}{2} \int \frac{d^{3} q_{1}}{(2 \pi)^{3} \tilde{r}\left(q_{1}\right)} \\
& \approx \tilde{r}\left(q_{c}\right)+u \frac{b}{\sqrt{\tilde{r}\left(q_{c}\right)}},
\end{aligned}
$$

where $b=q_{c} / 8 \pi$ and where we assumed

$$
q_{c}^{4} \gg r_{0}
$$

Now the expression $\left\langle\psi\left(\overrightarrow{q_{1}}\right) \psi\left(-\overrightarrow{q_{1}}+\vec{q}\right)\right\rangle$ must be found to all orders in loop expansion. We, however, are basically interested in the region in which the corrections for the correlations $\langle\psi(\vec{q}) \psi(-\vec{q})\rangle$ and for $\left\langle\psi(\vec{q}) \psi(-\vec{q}) \psi\left(\overrightarrow{q_{1}}\right) \psi\left(-\overrightarrow{q_{1}}\right)\right\rangle$ first become important. This occurs for the one-loop contribution in Eq. (24) and in the "ladder" diagrams. These diagrams have relative order

$$
u \int \frac{d^{3} q_{1}}{(2 \pi)^{3} \tilde{r}\left(q_{1}\right)^{2}} \approx u \frac{b}{\tilde{r}\left(q_{c}\right)^{3 / 2}},
$$

and they enter in all order in the loop expansion. In order to take them into account, we will assume that

$$
\frac{u q_{c}}{r_{0}^{3 / 2}} \approx 1
$$

Within this approximation, one can show that only ladder loop diagrams without dependence on the external momentum contribute to the averages in Eq. (23).

Using this and the condition Eq. (25), we eliminate all the complicated diagrams, and we end up with a self consistent form for the equation of state given by

$$
\begin{aligned}
h(\vec{q})= & {\left[r+\left(q^{2}-q_{c}^{2}\right)^{2}\right] \bar{\phi}(\vec{q})+\frac{w}{2} \int d^{3} q_{1} \bar{\phi}\left(\overrightarrow{q_{1}}\right) \bar{\phi}\left(\vec{q}-\overrightarrow{q_{1}}\right) } \\
& +\frac{u}{6} \iint d^{3} q_{1} d^{3} q_{2} \bar{\phi}\left(\overrightarrow{q_{1}}\right) \bar{\phi}\left(\overrightarrow{q_{2}}\right) \bar{\phi}\left(\vec{q}-\overrightarrow{q_{1}}-\overrightarrow{q_{2}}\right) \\
& -\frac{u}{2} \int d^{3} q_{1} \bar{\phi}\left(\overrightarrow{q_{1}}\right) \bar{\phi}\left(-\overrightarrow{q_{1}}\right) \bar{\phi}(\vec{q})
\end{aligned}
$$

where

$$
\begin{aligned}
r & =\tilde{r}+\frac{u\langle r\rangle}{2}+\frac{u}{2} \int d^{3} q_{1} \bar{\phi}\left(\overrightarrow{q_{1}}\right) \bar{\phi}\left(-\overrightarrow{q_{1}}\right), \\
\langle r\rangle & =\int \frac{d^{3} q_{1}}{(2 \pi)^{3} \tilde{r}\left(q_{1}\right)} .
\end{aligned}
$$

Now, in the nonuniform phase, the averages $\bar{\phi}\left(q=q_{c}\right)$ are nonzero, while higher harmonics of $\bar{\phi}(q)$ with $q \neq q_{c}$ are small. In view of that, we eliminate the noncritical terms in $h\left(q=q_{c}\right)=0$ in favor of the critical variables, obtaining finally a free energy depending only on $h(q=$ $q_{c}$ ) and given by

$$
h\left(q=q_{c}\right)=r_{l} a_{l}-\frac{u}{2} a_{l}^{3} .
$$

We assumed that

$$
\bar{\phi}=2 a_{l} \cos q_{c} z
$$

which specifies a lamellar phase. The parameters given by

$$
\begin{aligned}
& r_{l}=r_{0}+b \frac{u_{l}}{\sqrt{r_{l}}}+u_{l} a_{l}^{2} \\
& u_{l}=u-\frac{w^{2}}{r_{l}+q_{c}^{4}}
\end{aligned}
$$

contain contributions from the noncritical modes ( $b$ is a function of $q_{c}$ only).

For zero field, Eq. (30) leads to a nonzero amplitude

$$
a_{l}=\sqrt{\frac{2 r_{l}}{u}}
$$

and to a new expression for Eq. (32) given by

$$
-r_{0}=-\left(1-2 \delta_{l}\right) r_{l}+\frac{b u_{l}}{\sqrt{r_{l}}}
$$

where $\delta_{l} \equiv u_{l} / u$. This equation, which specifies the temperatures where this ordered phase can be found, has real solutions if

$$
-r_{0}>3\left(2 \delta_{l}-1\right)\left(\frac{b u_{l}}{2\left(2 \delta_{l}-1\right)}\right)^{2 / 3} .
$$

Now, in order to verify if the lamellar phase, specified by Eq. (33) and limited by the condition Eq. (35), is more favorable than the disordered phase, we look at the difference between the thermodynamic potentials associated with each one of those phases. This function, generated by integrating the equation of state, is given by

$$
\Delta F_{l}=\frac{r_{l}^{2}}{2 u_{l}}\left(1-2 \delta_{l}\right)-\frac{r_{1 l}^{2}}{2 u_{l}}+b \sqrt{r_{l}}-b \sqrt{r_{1 l}}
$$

where $r_{1 l}$ is solution of

$$
-r_{0}=-r_{1 l}+\frac{b u_{l}}{\sqrt{r_{1 l}}}
$$

The lamellar phase is, consequently, more favorable than the disordered phase if

$$
-r_{0}>2\left(2 \delta_{l}-1\right)\left(\frac{b u_{l}}{\left(2 \delta_{l}-1\right)}\right)^{2 / 3} .
$$

This result leads to the conclusion that a first-order phase boundary between those phases is possible. We still need, however, to verify if no other modulated phases can be present.

The other type of modulated phase that one can find in such a system is the hexagonal phase, specified by 


$$
\begin{aligned}
\bar{\phi}= & 2 a_{h}\left[\cos \left(q_{c} z\right)+\cos \left(\frac{\sqrt{3}}{2} q_{c} y-\frac{1}{2} q_{c} z\right)\right. \\
& \left.+\cos \left(\frac{\sqrt{3}}{2} q_{c} y+\frac{1}{2} q_{c} z\right)\right]
\end{aligned}
$$

Following similar steps to those we use with the lamellar phase, we found that the hexagonal phase has an equation of state given by

$$
h\left(q=q_{c}\right)=r_{h} a_{h}+w a_{h}^{2}-\frac{u}{2} a_{h}^{3}
$$

where

$$
\begin{aligned}
& r_{h}=r_{0}+b \frac{u_{h}}{\sqrt{r_{h}}}+3 u_{h} a_{h}^{2} \\
& u_{h}=u-\frac{w^{2}}{r_{h}+q_{c}^{4}}
\end{aligned}
$$

and where, for $a_{h}=0$, one has

$$
-r_{0}=-r_{1 h}+\frac{b u_{h}}{\sqrt{r_{1 h}}}
$$

This equation always has a real solution that specifies the hexagonal phase. Such a phase is more favorable than the disordered phase if the free energy given by
$\Delta F_{h}=\frac{r_{h}^{2}}{2 u_{h}}-\frac{r_{1 h}^{2}}{2 u_{h}}+b \sqrt{r_{h}}-b \sqrt{r_{1 h}}+2 w a_{h}^{3}-\frac{3}{4} u a_{h}^{4}$

is negative.

Now we must check which modulated phase is more favorable. In order to do it, we compare the free energies Eq. (36) and Eq. (43) and we obtain a phase diagram in terms of the physical parameters: temperature $T$, the external field $h$, and the bending energy $\kappa$, as follows (see Fig. 6). For small values of the field $h<h_{t}$, the lamellar phase dominates over the hexagonal phase. For $h>h_{t}$, or otherwise at high temperatures the hexagonal phase coexists with the disordered phase. As the temperature is decreased, one finds a phase boundary between the hexagonal and lamellar phases. At $h=h_{t}$, the three phases coexist at a triple point. On varying $\kappa$, one generates a triple line, $h=h_{t}$. This line is an increasing function of the critical wave vector. In that sense, we can say that even at the critical wave vector the triple point exists and conversely the first-order phase boundary between the disordered and lamellar phases. Therefore the mean-field Lifshitz point is actually a fluctuation-induced end point.

\section{ACKNOWLEDGMENTS}

This work was supported in part by CNPq-Conselho Nacional de Desenvolvimento Científico e Tecnológico and FINEP - Financiadora de Estudos e Projetos, Brazil.
[1] For a review, see K. A. Dawson, in Structure and Dynamics of Strongly Interacting Colloids and Supramolecular Aggregates in Solution, edited by S.-H. Chen et al. (Kluwer Academic Publishers, Netherlands, 1992).

[2] H. T. Davis, J. F. Bodet, L. E. Scriven, and W. G. Miller, in Physics of Amphiphilic Layers, edited by J. Meunier, D. Langevin, and N. Bociara, Spring-Verlag Proceedings in Physics Vol. 21 (Springer-Verlag, Berlin, 1987).

[3] T. A. Witten, Phys. Today 43, 21 (1990).

[4] S. -H. Chen, S. L. Chang, R. Strey, J. Samseth, and K. Mortensen, J. Phys. Chem. 95, 7427 (1991).

[5] S. J. Chen, D. F. Evans, B. W. Ninham, D. J. Mitchell, F. D. Blum, and S. Pickup, J. Chem. Phys. 90, 842 (1986); U. Olsson, K. Shinoda, and B. Lindman, ibid. 90, 4083 (1986).

[6] S. Bhattacharya, J. P. Stokes, M. W. Kim, and J. S. Huang, Phys. Rev. Lett. 55, 1884 (1985); M. W. Kim and J. S. Huang, Phys. Rev. A 34, 719 (1986); C. Cametti et al., Phys. Rev. Lett. 64, 1461 (1990).

[7] B. Widom, J. Chem. Phys. 84, 6943 (1986); K. A. Dawson, M. D. Lipkin, and B. Widom, ibid. 88, 5149 (1988), B. Widom, M. D. Lipkin, and K. A. Dawson, Physica A 140, 26 (1986); K. A. Dawson, Phys. Rev. A 36, 3383 (1987); J. C. Wheeler and B. Widom, J. Am. Chem. Soc. 90, 3064 (1968).

[8] G. Gompper and M. Schick, Phys. Rev. B 41, 9148 (1990).
[9] Per A. Slotte, Phys. Rev. B 46, 6469 (1992).

[10] A. Ciach, J. S. Hoye, and G. Stell, J. Chem. Phys. 90, 1214 (1989).

[11] J. R. Gunn and K. A. Dawson, J. Chem. Phys. 96, 3152 (1992).

[12] P. J. Upton and J. M. Yeomans, Europhys. Lett. 5, 575 (1988); Phys. Rev. B 40, 479 (1989).

[13] K. A. Dawson, Phys. Rev. A 36, 3383 (1987).

[14] Marcia C. Barbosa, Phys. Rev. B 42, 6363 (1990).

[15] Y. Levin and K. A. Dawson, Phys. Rev. A 42, 1976 (1990).

[16] Marcia C. Barbosa, Phys. Rev. B 46, 3672 (1992).

[17] Y. Levin, C. J. Mundy, and K. A. Dawson, Phys. Rev. A 45, 7309 (1992).

[18] C. S. O. Yokoi, M. D. Coutinho-Filho, and S. R. Salinas, Phys. Rev. B 31, 4502 (1985).

[19] Marcia C. Barbosa, Phys. Rev. E 48, 1744 (1993).

[20] Figure 4(c) is a highly schematic phase diagram. More precise results were obtained in Ref. [19] for regions far from the Lifshitz point and, consequently, we cannot be sure that the first-order line that separates the disordered phase from the modulated phase and the continuous line share the same tangent, as should be expected for the usual tricritical points.

[21] S. A. Brazovskii, Zh. Eksp. Teor. Fiz. 68, 175 (1975) [Sov. Phys. JETP 41, 85 (1975)]. 